\title{
COMPRESSIVE BEHAVIOR FOR SURFACE-NANOCRYSTALLIZED AL-ALLOY MATERIAL
}

Yueguang Wei, Chen Zhu, Xiaolei Wu

LNM, Institute of Mechanics, Chinese Academy of Sciences, Beijing 100080, P.R. China

\begin{abstract}
Mechanical behavior of the surface-nanocrystallized material fabricated by the ultrasonic shot peening method is investigated experimentally and theoretically. In the experimental research, based on microscopic observations, the compressive specimens are designed and machined considering the material features, and the compressive experiments are performed. Furthermore the compressive stress-strain curves are measured. In the theoretical research, a microstructure cell model is presented considering the material microscopic organization observed from experiments, and the compressive stress-strain relations are simulated. Both experimental and theoretical results display the strong size effect on the material behavior.
\end{abstract}

\section{INTRODUCTION}

Recent researches have shown that the high-quality nano-structured materials can be fabricated by some advanced techniques, and the some conventional materials can be improved in quality by a surface-nanocrystalline technique. For example, using so called the severe plastic deformation (SPD) method, the nanocrystalline material has been fabricated [1-5]. The adopted severe plastic deformation method mainly includes the large torsion [1], large pressing [4] and the ultrasonic shot peening [5], etc. The advantages of the nano-structured materials and the surface-nanocrystallized materials are displayed with material regular microstructures globally or locally. The size of the microstructure is measured from several ten nanometers to several hundred nanometers even to several microns. Within the length scales, materials usually behave strong size effect. On the research of the size effect, many investigations have been performed for the micro-indentation tests of the single crystal metals previously. Through theoretical and experimental researches for the micro-indentation test, one found that with decreasing applied load, or with decreasing the indentation depth, the obtained hardness curve displayed a going up trend, i.e., the size effect. The size effect could be described using the strain gradient theories [6-9], and the prediction results were consistent with the experimental measurement. The research showed that the microscale length parameter for the single crystal metal materials was taken in an order of microns [10,11]. However, the case is complicated for a nano-crystallized material, about which besides the above size effect, basically the influences of the crystal grain size and its shape distribution, i.e., the effect of the micro-structure features on the material behavior must be considered. In author's previous research for systems of the nano-polycrystal Al and the thin film/substrate [12], the effects of the crystal grain size and its shape distribution were called geometrical effects, for differing from the size effect. Based on the microstructure cell model and the strain gradient plasticity, the size effect and geometrical effect (when the microstructure size was in micron or sub-micron order) have been studied [12].

In the present research, the mechanical behavior of the surface-nanocrystallized Al-alloy 
material (SNCAA) fabricated by the ultrasonic shot peening method [5] will be studied experimentally and theoretically. In experimental research, test specimen is designed and prepared according to microstructure features, and compressive experiments will be performed. The compressive stress-strain curves are measured. In theoretical research, simplified mechanics model will be presented and discussed in detail, and a microstructure cell model is developed to describe the material behavior. Using the cell model and the strain gradient plasticity theory, the compressive stress-strain relations with related model and material parameters will be analyzed. During simulation, some parameter values are come from experimental measurements, and others are determined through comparing experimental result with theoretical result.

\section{MICROSCOPIC OBSERVATION AND ANALYSIS FOR THE SNCAA MATERIAL}

Photos of the conventional and high-resolution electron microscopy and TEM about the SNCAA fabricated by the ultrasonic shot peening show in Figure 1. The material fabrication was carried out in the Metal Material Institute, Chinese Academy of Sciences [13]. The nanocrystalline principle is sketched in Figure 1(a). Figure 1(b) shows TEM photo of the distribution of nano-crystallized grains. From Figure 1(b), the most grain size is in an order of about several ten of nano-meters. Basically, the distribution of the grain size is uniform locally. Figure 1(c) shows photo of the high-resolution electron microscopy about the nano-crystal grain boundary. Clearly, the grain boundary region is in an orderly structure. Figure 1(d) is another high-resolution photo of electron microscopy, shows the distributed dislocations in a nano-crystal. Using these microscopic observations, one obtains a basic understanding to the SNCAA material.

\section{COMPRESSIVE EXPERIMENTAL STRESS-STRAIN CURVES}

It is an expectation that the specimen design and preparation for a compressive test can basically display the mechanical behavior for the surface-nanocrystallized material. For this reason, two types of specimens are designed, which are the fully influenced specimens by nano-crystallization, and the uninfluenced specimens in the nano-crystallization, respectively. Two type specimens were cut in an order respectively from nanocrystalline surface toward inside the material, as shown in Figure 2. Since each specimen is a cubic column, $1 \mathrm{~mm} \times 1 \mathrm{~mm} \times 2 \mathrm{~mm}$, so that the second kind specimens are nearly uninfluenced by the nanocrystalline procedure. After each specimen was cut, it was ground and polished with number 600 1200 sand sheets smoothly. Each type consists of 6 specimens. Compressive experiments were performed at the Laboratory of the Mechanics and Engineering Department, Peking University. During the compressive experiments, displacement loading was adopted. Figure 3 shows the compressive stress-strain curves, measured from experiments based on the above mentioned two type specimens. Each type has 6 specimens, and the measured curves have the small differences from Figure 3. The compressive stress-strain curves obtained from the SNCAA specimens are clearly higher than those of uninfluenced specimens.

\section{COMPRESSIVE STRESS-STRAIN RELATIONS, SIMULATION RESULTS}


Surface-Nanocrystallized Material

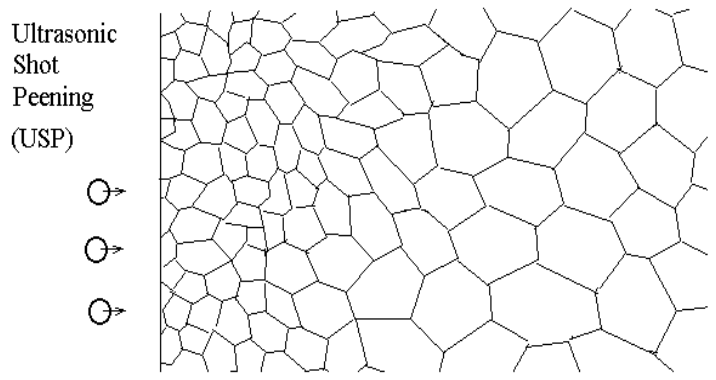

(a)

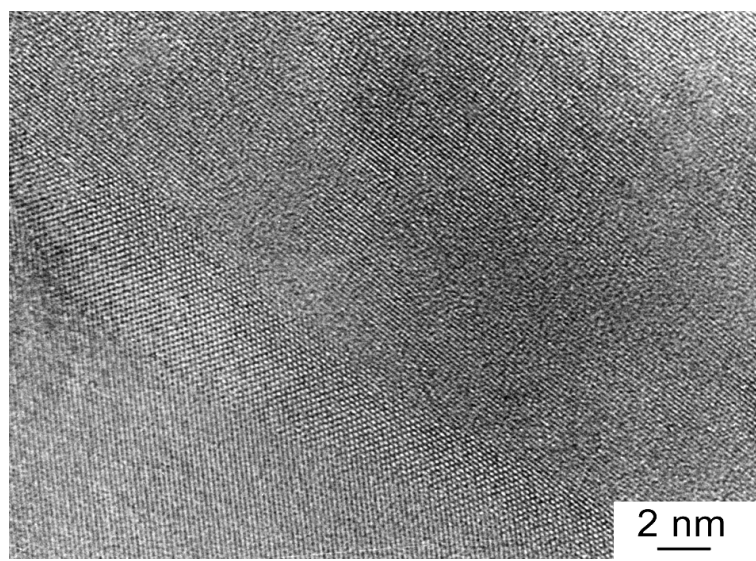

(c)

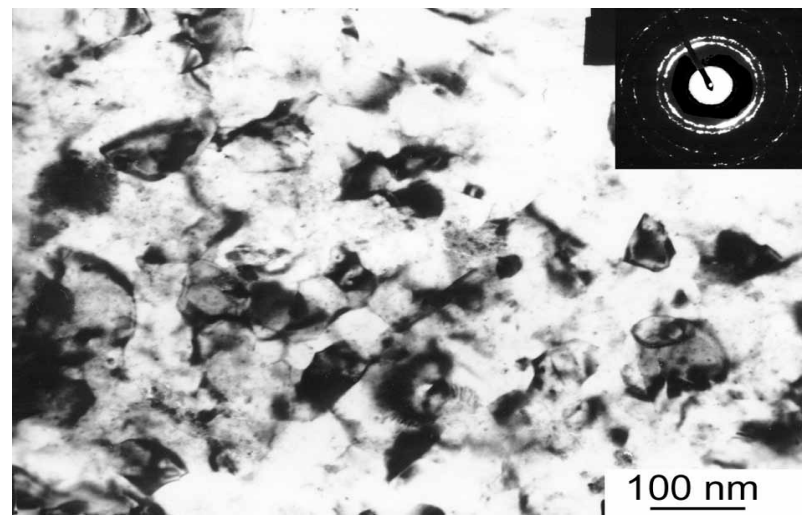

(b)

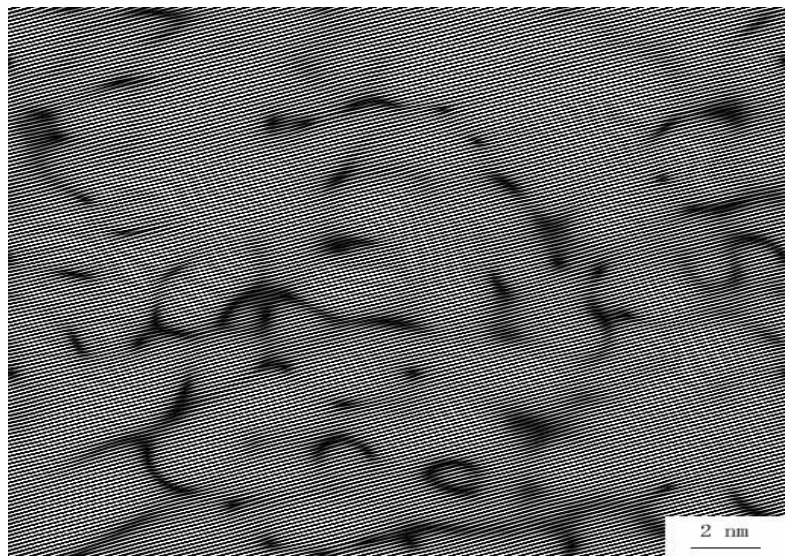

(d)

Figure 1. Microscopic organization features of the surface-nanocrystallized Al-alloy material. (a) Surface-nanocrystalline method-ultrasonic shot peening method, (b) the formed new grains, (c) the profile of the grain boundary zone, and (d) the formed dislocations within a grain.

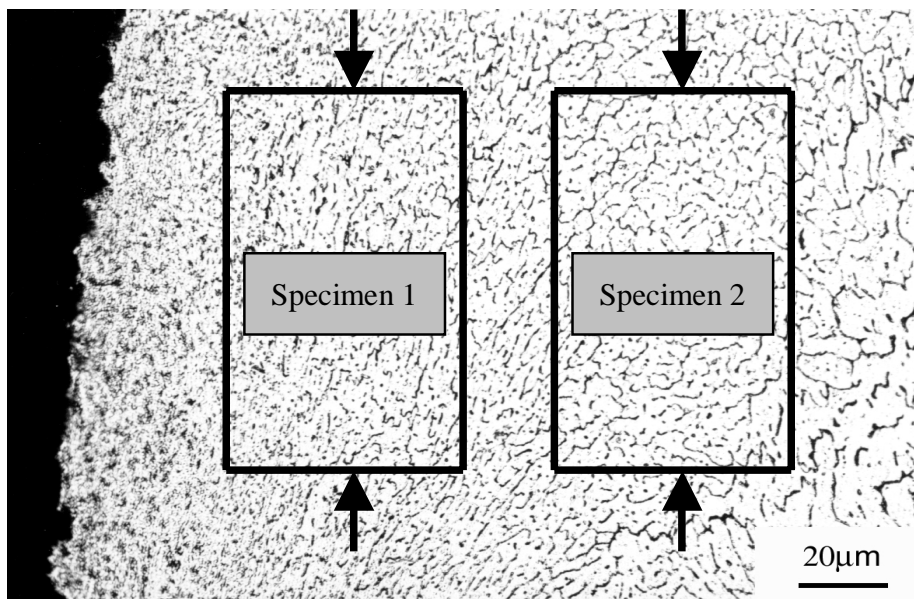

Figure 2. The specimen design for compressive experiment 


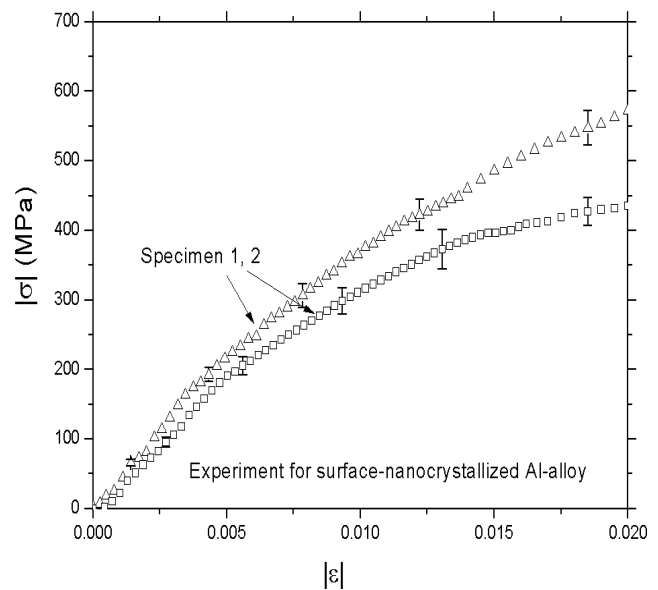

Figure 3. Compressive stress-strain curves of two types of specimens, influenced and uninfluenced specimens by nanocrystalline

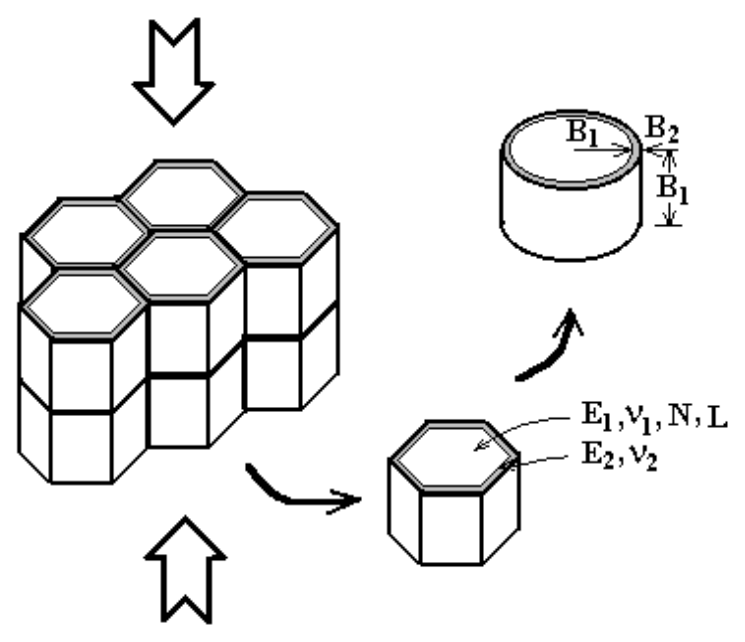

Figure 4. The microstructure cell model of compressive experiment for nano-crystallized material

The material size effect can not be predicted from conventional elastic-plastic theory. Therefore, we use the strain gradient plasticity theory[6] combined with a microstructure cell model considering the compressive test feature to simulate the compressive stress-strain relations of the SNCAA material. The foundalmental relations of Fleck-Hutchinson strain gradient plasticity theory can be referred to literatures $[6,7,11,12]$. Comparing with conventional elastic-plastic theory, the strain gradient plasticity theory includes an additional length parameter $\mathrm{L}$, which can be used to characterize the strain gradient effect. In macroscale, the strain gradient effect is much weak so that it can be neglected, however in the microscale (specifically in the order of micron or sub-micron), the strain gradient effect is important. The length parameter $\mathrm{L}$ is sometimes called micro-scale parameter.

By examining the microstructure characteristics of the SNCAA material shown in Figure 1(b) (d) and in Figure 2 (specimen orientation and location), a microstructure cell model for compressive test is presented and shown in Figure 4. Grain shape is simplified as a six-edge column, and distributed inside material periodically. Each column is connected with another by a thin layer material, which characterizes the grain boundary layer. For further simplicity, the grain shape is simplified as a cylinder. Effective radius and height of a representative grain is described by $\mathrm{B}_{1}$. Thickness of the grain boundary layer is denoted by $2 \mathrm{~B}_{2}$. Through microscopic observation and analysis of the SNCAA material, during nano-crystallization process, the original large grains were broken down into many small grains, and inside the grain a large number of dislocations were produced and moved to gather around the new grain boundaries. Therefore, in the simplified model, grain is taken as an elastic-plastic material, and characterized by the strain gradient plasticity theory, while grain boundary layer is taken as an elastic material 
and characterized by conventional elastic theory. The related parameters for grain material are $\mathrm{E}_{1}$ (Young's modulus), $v_{1}$ (Poisson ratio), $\mathrm{N}$ (strain hardening exponent) and L (length scale parameter), while the related parameters for grain boundary layer are $E_{2}$ and $v_{2}$. The size ratio of the elastic-plastic material zone with the elastic material zone is $\mathrm{B}_{1} / \mathrm{B}_{2}$, which can be used to characterize the volume fractions of the grain region and the grain boundary region.

The compressive stress-strain relation with independent parameters can be expressed as

$$
\frac{|\sigma|}{\sigma_{Y}}=f\left(|\varepsilon| ; \frac{E_{1}}{\sigma_{Y}}, \frac{E_{2}}{E_{1}}, \nu_{1}, v_{2}, N, \frac{B_{2}}{B_{1}}, \frac{L}{B_{1}}\right)
$$

One can carry out the finite element simulation for the compressive experiments using the microstructure cell model (see Figure 4) and the strain gradient plasticity theory. For the axisymmetrical simplified model shown in Figure 4, nine-node isoparametric displacement element with $2 \times 2$ Gauss points is adopted. Figure 5 shows the simulated compressive stress-strain curves with several cell sizes and several material parameters. Considering the metal material, some material parameters taken in the simulation are shown in Figure 5. On the model parameter $\mathrm{B}_{1} / \mathrm{B}_{2}$ value, from the observation of microscopic organization of SNCAA it is about $0.03 \sim 0.06$, therefore in the simulation it is taken as 0.03 and 0.06 , respectively. Figure 5 shows the compressive stress-strain curves for several values of $\mathrm{L} / \mathrm{B}_{1}$. When grain size is very large (or $\mathrm{L} / \mathrm{B}_{1}$ is very small), the compressive stress-strain curve is very low. However, with decreasing the grain size it goes up. Furthermore, when grain size decreases until it is comparable with material length parameter L, to continue decreasing the grain size has only a small influence on the material strength (or stress-strain relations).

Figure 6 shows both comparisons of the simulated with average experimental stress-strain relations, for $\mathrm{L}=0.5$ micron and $\mathrm{B}_{1}=0.1$ and 100 microns respectively. From Figure 6 , the prediction results and the experimental results are basically consistent with each other for the proper selections of model parameters and material parameters.

\section{CONCLUSION}

A system study for the surface-nanocrystallized Al-alloy has been performed in this paper. The research includes the following contents. Mechanical behavior of the surface-nanocrystallized material fabricated by using the ultrasonic shot peening method has been investigated experimentally and theoretically. In the experimental research, high-resolution electron microscopy observations are performed, and based on the observation, the compressive specimens were designed and machined considering the material features. The compressive experiments have been carried out, and the compressive stress-strain curves have been measured. In the theoretical research, a microstructure cell model has been presented considering the material microscopic organization features observed from experiments, and the compressive stress-strain relations have been simulated. Both experimental and theoretical results have shown the strong size effect on the material behavior. 


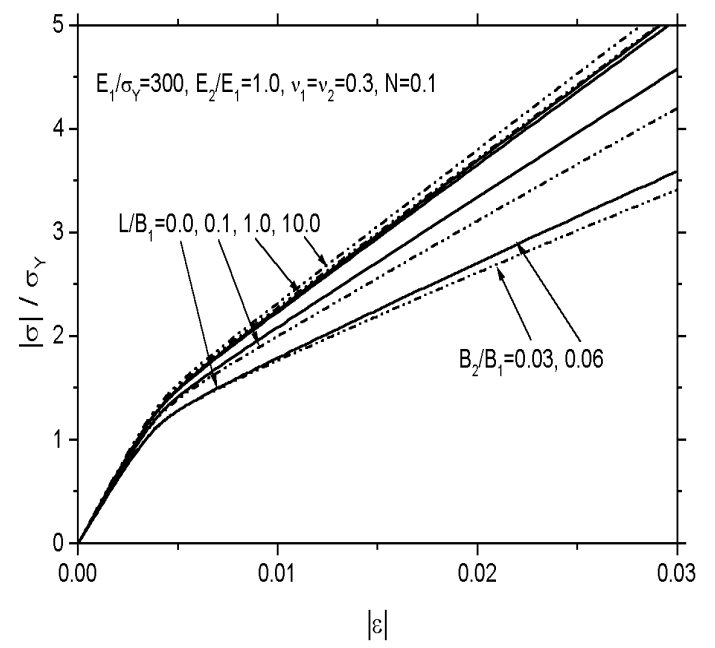

Figure 5. The simulated compressive stress-strain relations for several model parameters

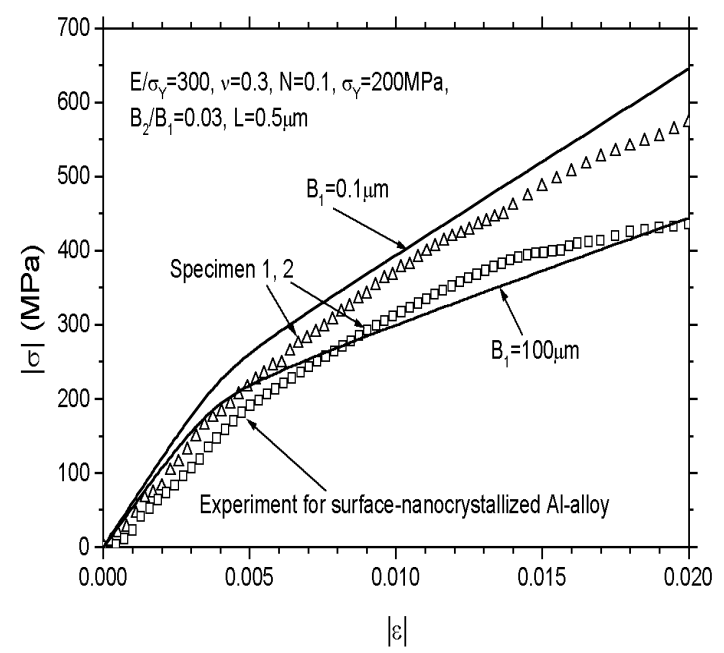

Figure 6. The comparison of compressive stress-strain relations from experiment and simulation

\section{ACKNOWLEDGMENTS}

This work was supported by the National Natural Science Foundation of China through Grant 19925211, and jointly supported by the Chinese Academy of Sciences through "Bai Ren Plan".

\section{REFERENCES}

1 Valiev RZ, Korznikov AV, Mulyukov RR. Mater. Sci. Eng., A168, 141(1993).

2 Valiev RZ, Ivanisenko YV, Rauch EF, Baudelet B. Acta Mater., 44, 4705 (1996).

3 Valiev RZ, Islamgaliev RK. Mater. Sci. Forum, 304, 39 (1999).

4 Valiev RZ, Islamgaliev RK, Alexandrov IV. Prog. in Mater. Sci., 45, 103 (2000).

5 Lu K, Lu J. J. Mater. Sci \& Tech, 15, 193 (1999).

6 Fleck NA, Hutchinson JW. Adv. in Appl. Mech., 33, 295 (1997).

7 Wei Y, Hutchinson JW. J. Mech. Phys. Solids, 45, 1253 (1997).

8 Gao H, Huang Y, Nix W D, Hutchinson J W. J. Mech. Phys. Solids, 47, 1239 (1999).

9 Chen S, Wang TC. Acta Mater., 48, 3997 (2000).

10 Begley M, Hutchinson J W. J. Mech. Phys. Solids, 46, 1029 (1998).

11 Wei Y, Wang X, Wu X, Bai Y. Science in China (Series A), 44, 74 (2001).

12 Wei Y, Wang X, Zhao M, Cheng CM, Bai Y. Acta Mechanica Sinica, 19, No.1, (2003).

13 Zhu C. Master Thesis, Institute of Mechanics, Chinese Academy of Sciences, June, (2002). 